\title{
The electronic structure of bispidine iron(IV) oxo complexes
}

Anna E. Anastasi ${ }^{a)}$, Peter Comba $^{b)^{*}}$, John McGrady ${ }^{c)}$, Achim Lienke ${ }^{d)}$ and Heidi Rohwer ${ }^{b)}$

a) Department of Chemistry, University of York, Heslington, York YO10 5DD

b) Anorganisch-Chemisches Institut, Universität Heidelberg, Im Neuenheimer Feld 270, 69120 Heidelberg, fax: +49 6221 546617, e-mail: peter.comba@aci.uni-heidelberg.de

c) WestCHEM, Department of Chemistry, University of Glasgow, Glasgow, G12 8QQ, e-mail: j.mcgrady@chem.gla.ac.uk

d) Unilever Research Vlaardingen, Olivier van Noortlaan 120, 3133 AT Vlaardingen, The Netherlands, e-mail: achim.lienke@unilever.com

\section{Supplementary Material}


Table S1: Bond lengths, Mulliken spin densities, absolute and relative energies and triplet-quartet splittings for the bispidine $\mathrm{Fe}^{\mathrm{IV}}=\mathrm{O}$ complexes. Relative energies are given with respect to the most stable spin state of a particular complex, regardless of geometry. Triplet-quintet splittings are given for each isomer.

\begin{tabular}{|c|c|c|c|c|c|c|c|c|c|c|c|c|c|c|c|c|}
\hline & & & \multicolumn{5}{|c|}{ Bond lengths / $\AA$} & \multicolumn{6}{|c|}{ Mulliken spin densities } & \multirow{2}{*}{$\begin{array}{c}\text { Energy } \\
\text { [a.u] }\end{array}$} & \multirow{2}{*}{$\frac{\mathrm{E}_{\mathrm{rel}}}{\left[\mathrm{kJ} \mathrm{mol}^{-1}\right]}$} & \multirow{2}{*}{$\frac{\Delta \mathrm{E}_{\mathrm{T} \rightarrow \mathrm{Q}}}{\left[\mathrm{kJ} \mathrm{mol}^{-1}\right]}$} \\
\hline & $\mathrm{Fe}=\mathrm{O}$ & $\mathrm{s}$ & $\mathrm{Fe}-\mathrm{O}$ & Fe-N7 & $\mathrm{Fe}-\mathrm{N3}$ & Fe-Npy & $\mathrm{Fe}-\mathrm{X}$ & $\mathrm{Fe}$ & O & N7 & N3 & Npy & $x$ & & & \\
\hline \multicolumn{17}{|c|}{ 5-coordinate complexes, $\left[\left(\mathrm{L}^{1,2}\right) \mathrm{Fe}^{\mathrm{IV}}=0\right]^{2+}$} \\
\hline \multirow[t]{2}{*}[(\mathrm{L}^{1})\mathrm{Fe}\mathrm{IV}^{\mathrm{IV}}=\mathrm{O}]{$^{2+}$} & trans-N3 & 1 & 1.579 & 2.040 & 2.030 & 2.000 & - & 1.62 & 0.47 & -0.05 & -0.02 & -0.02 & - & -2369.6141 & +24.9 & \multirow{3}{*}{-24.9} \\
\hline & & 2 & 1.614 & 2.184 & 2.056 & 2.002 & - & 3.11 & 0.70 & 0.12 & -0.03 & 0.00 & - & -2369.6236 & 0.0 & \\
\hline \multirow[t]{3}{*}[(\mathrm{L}^{2})\mathrm{Fe}^{\mathrm{IV}}=\mathrm{O}]{$^{2+}$} & trans-N7 & 1 & 1.579 & 2.146 & 1.952 & 2.022 & - & 1.59 & 0.50 & -0.01 & -0.05 & -0.02 & - & -2448.2351 & +28.8 & \\
\hline & & 2 & 1.616 & 2.168 & 2.129 & 2.042 & - & 3.15 & 0.70 & -0.02 & 0.15 & -0.01 & - & -2448.2308 & +40.1 & \multirow[t]{2}{*}{+11.3} \\
\hline & trans-N3 & 2 & 1.613 & 2.179 & 2.047 & 2.054 & - & 3.11 & 0.72 & 0.10 & -0.04 & 0.01 & - & -2448.2461 & 0.0 & \\
\hline \multicolumn{17}{|c|}{ 6-coordinate complexes, $\left[(\mathrm{X})\left(\mathrm{L}^{1,2}\right) \mathrm{Fe}^{\mathrm{IV}}=0\right]^{2+}$} \\
\hline \multirow[t]{4}{*}[(\mathrm{OH}_{2})(\mathrm{L}^{1})\mathrm{Fe}^{\mathrm{IV}}=\mathrm{O}]{$^{2+}$} & trans-N3 & 1 & 1.617 & 2.099 & 2.100 & 1.987 & 2.069 & 1.28 & 0.80 & -0.04 & -0.02 & -0.01 & -0.01 & -2446.0577 & +7.1 & \multirow{2}{*}{-7.1} \\
\hline & & 2 & 1.615 & 2.281 & 2.082 & 2.030 & 2.335 & 3.15 & 0.67 & 0.11 & -0.04 & 0.01 & 0.02 & -2446.0604 & 0.0 & \\
\hline & trans-N7 & 1 & 1.612 & 2.270 & 1.970 & 2.000 & 2.084 & 1.23 & 0.80 & -0.02 & -0.04 & -0.01 & 0.00 & -2446.0519 & +22.3 & \multirow{2}{*}{+9.4} \\
\hline & & 2 & 1.612 & 2.230 & 2.167 & 2.090 & 2.177 & 3.19 & 0.63 & -0.03 & 0.11 & 0.03 & 0.02 & -2446.0483 & +31.8 & \\
\hline \multirow[t]{4}{*}[(\mathrm{NCCH}_{3})(\mathrm{L}^{1})\mathrm{Fe}^{\mathrm{IV}}=\mathrm{O}]{$^{2+}$} & trans-N3 & 1 & 1.621 & 2.137 & 2.096 & 1.985 & 1.991 & 1.22 & 0.86 & -0.02 & -0.02 & -0.01 & 0.00 & -2502.3961 & 0.0 & \multirow{2}{*}{+4.2} \\
\hline & & 2 & 1.613 & 2.321 & 2.097 & 2.036 & 2.302 & 3.13 & 0.68 & 0.09 & -0.04 & 0.02 & 0.04 & -2502.3945 & +4.2 & \\
\hline & trans-N7 & 1 & 1.617 & 2.309 & 1.992 & 2.000 & 1.991 & 1.23 & 0.87 & -0.02 & -0.03 & -0.01 & 0.01 & -2502.3910 & +13.4 & \multirow{2}{*}{+22.1} \\
\hline & & 2 & 1.611 & 2.257 & 2.181 & 2.107 & 2.165 & 3.16 & 0.66 & -0.03 & 0.09 & 0.04 & 0.02 & -2502.3826 & +35.5 & \\
\hline
\end{tabular}




\begin{tabular}{|c|c|c|c|c|c|c|c|c|c|c|c|c|c|c|c|c|}
\hline \multirow[t]{4}{*}[(\text{pyridine})(\mathrm{L}^{1})\mathrm{Fe}^{\mathrm{IV}}=\mathrm{O}]{$^{2+}$} & trans-N3 & 1 & 1.616 & 2.200 & 2.124 & 1.990 & 2.088 & 1.28 & 0.81 & -0.02 & -0.02 & -0.01 & -0.02 & -2617.8961 & 0.0 & \multirow{2}{*}{+3.4} \\
\hline & & 2 & 1.610 & 2.360 & 2.113 & 2.063 & 2.307 & 3.15 & 0.64 & 0.08 & -0.03 & 0.02 & 0.06 & -2617.8948 & +3.4 & \\
\hline & trans-N7 & 1 & 1.609 & 2.451 & 2.021 & 2.015 & 2.065 & 1.30 & 0.80 & -0.02 & -0.02 & -0.01 & -0.01 & -2617.8901 & +15.8 & \\
\hline & & 2 & 1.609 & 2.287 & 2.180 & 2.185 & 2.152 & 3.20 & 0.62 & -0.03 & 0.07 & 0.06 & 0.03 & -2617.8854 & +28.1 & \\
\hline \multirow[t]{4}{*}[(\mathrm{OH})(\mathrm{L}^{1})\mathrm{Fe}^{\mathrm{IV}}=\mathrm{O}]{$^{+}$} & trans-N3 & 1 & 1.616 & 2.275 & 2.122 & 1.982 & 1.852 & 1.44 & 0.66 & 0.00 & -0.01 & -0.01 & -0.08 & -2445.7690 & +18.6 & \multirow{2}{*}{-18.1} \\
\hline & & 2 & 1.615 & 2.332 & 2.188 & 2.207 & 1.809 & 3.22 & 0.50 & 0.01 & -0.01 & 0.06 & 0.13 & -2445.7759 & +0.5 & \\
\hline & trans-N7 & 1 & 1.615 & 2.067 & 2.364 & 1.981 & 1.852 & 1.41 & 0.70 & -0.01 & 0.00 & -0.01 & -0.06 & -2445.7675 & +22.6 & \multirow{4}{*}{-7.4} \\
\hline & & 2 & 1.608 & 2.303 & 2.211 & 2.221 & 1.803 & 3.15 & 0.53 & -0.02 & 0.02 & 0.06 & 0.20 & -2445.7761 & 0.0 & \\
\hline \multirow[t]{2}{*}{$\left(\mathrm{NCCH}_{3}\right)\left(\mathrm{L}^{2}\right) \mathrm{Fe}^{\mathrm{IV}}=\mathrm{O}^{2+}$} & trans-N3 & 1 & 1.618 & 2.144 & 2.088 & 2.048 & 1.988 & 1.18 & 0.89 & -0.03 & -0.02 & -0.01 & 0.00 & -2581.0118 & +7.4 & \\
\hline & & 2 & 1.611 & 2.263 & 2.080 & 2.118 & 2.298 & 3.13 & 0.69 & 0.08 & -0.04 & 0.03 & 0.04 & -2581.0146 & 0.0 & \\
\hline \multicolumn{17}{|c|}{ 6-coordinate complexes, $\left[\left(\mathrm{L}^{3.4}\right) \mathrm{Fe}^{\mathrm{IV}}=0\right]^{2+}$} \\
\hline \multirow[t]{2}{*}[(\mathrm{L}^{4})\mathrm{Fe}\mathrm{IV}^{\mathrm{IV}}=\mathrm{O}]{$^{2+}$} & trans-N3 & 1 & 1.619 & 2.143 & 2.069 & 1.988 & 1.980 & 1.28 & 0.80 & -0.01 & -0.02 & -0.01 & -0.02 & -2616.6933 & 0.0 & \multirow{2}{*}{+26.5} \\
\hline & & 2 & 1.610 & 2.236 & 2.093 & 2.125 & 2.089 & 3.16 & 0.61 & 0.05 & -0.03 & 0.05 & 0.05 & -2616.6832 & +26.6 & \\
\hline \multirow[t]{2}{*}[(\mathrm{L}^{3})\mathrm{Fe}^{\mathrm{IV}}=\mathrm{O}]{$^{2+}$} & trans-N7 & 1 & 1.616 & 2.225 & 2.004 & 2.004 & 1.988 & 1.27 & 0.81 & -0.02 & -0.02 & -0.01 & -0.01 & -2616.6896 & +9.7 & \multirow{2}{*}{+22.6} \\
\hline & & 2 & 1.610 & 2.206 & 2.189 & 2.189 & 2.059 & 3.17 & 0.60 & -0.03 & 0.07 & 0.06 & 0.03 & -2616.6810 & +32.4 & \\
\hline
\end{tabular}

